\title{
SOBRE LAS AGUAS MINERALES NATURALES DE ESPAÑA: ASOCIACIONES ENTRE SU COMPOSICIÓN QUÍMICA Y LOCALIZACIÓN GEOGRÁFICA
}

\author{
J. MARTÍN-GIL, P. MARTÍN-RAMOS y F. J. MARTÍN-GIL \\ Departamento de Ingeniería Agrícola y Forestal \\ Escuela Técnica Superior de Ingenierías Agrarias. Palencia
}

\begin{abstract}
Resumen: Un estudio comparativo de las aguas minerales naturales de España, realizado a través de la composición de las aguas envasadas, nos ha llevado a advertir una serie de observaciones que ponen en relación dichas composiciones con la situación geográfica de los manantiales. De especial interés son las asociaciones latitud-salinidad y longitud-mineralización, no referidas anteriormente.
\end{abstract}

Palabras clave: aguas minerales naturales.

Summary: A comparative study of the natural mineral waters of Spain, accomplished through the chemical composition of the packed waters, it has carried us to warn a series of observations that put on relationship the composition of the water with the geographical situation of the springs. Of special interest are the latitude-salinity and longitude-mineralization associations, not reported previously

Key words: natural spring waters.

\section{INTRODUCCIÓN}

Las aguas minerales y termales constituyen un recurso geológico aprovechable como aguas envasadas comercialmente, o con fines terapéuticos y de descanso en balnearios. El número de manantiales registrados en España es algo superior a 2.000. De éstos, medio centenar ha dado lugar a plantas de embotellamiento de agua y un centenar, a estaciones termales activas. La legislación actual distingue entre distintos tipos de aguas: las de consumo público envasadas, las aguas preparadas, las aguas de manantial y las aguas minerales naturales. Las aguas minerales naturales deben cumplir, entre otros requerimientos, 
los de mantener estable su composición química, el caudal de su manantial, y la temperatura de surgencia. Estas condiciones son cumplidas prácticamente por casi la totalidad de las aguas estudiadas. Adicionalmente, la tercera parte de las aguas minerales naturales de España ha sido declarada minero-medicinal.

\section{MATERIAL Y MÉTODOS}

Los análisis químicos que han servido para la realización del estudio, y que figuran en las etiquetas de los envases, proceden fundamentalmente de siete laboratorios: el del Dr. Oliver Rodés, de Barcelona (acreditado como principal laboratorio de referencia en materia de aguas minerales); el del Instituto Tecnológico GeoMinero de España, de Madrid (aguas de Carrizal, Peñaclara, Fontoira y Corconte); el del Centro Nacional de Alimentación y Nutrición (Bezoya); el del AINIA, Paterna, Valencia (Fontsol); el de la sección de Minas de la Delegación Provincial del Ministerio de Industria en Zaragoza (Veri); el del Lcdo. M. A. Valero, de Zaragoza (El Cañar); y el del Laboratorio LANYN, de Pamplona (Uralai). En el caso de las aguas de Zambra y Viladrau, los análisis proceden de los laboratorios de análisis de las propias plantas envasadoras. Generalmente, la composición química de las aguas es controlada por los laboratorios de tales plantas embotelladoras.

\section{RESULTADOS}

Los principales componentes químicos de las aguas estudiadas aparecen en la Tabla 1. En ella se recogen los contenidos en bicarbonatos, sulfatos, cloruros, nitratos, sodio, potasio, calcio, magnesio y dióxido de silicio, expresados en $\mathrm{mg} / \mathrm{L}$. Otros componentes de difusión limitada o minoritarios, como es el caso de los iones fluoruro (solo frecuentes en los manantiales de Galicia, Asturias y algunos de La Rioja y Cataluña, y que solo alcanzan cantidades significativas en el agua Fontecelta $(10 \mathrm{mg} / \mathrm{L}))$, no han sido objeto de consideración.

El análisis comparativo de los datos de la Tabla 1 permite establecer las siguientes deducciones:

1. Las aguas menos mineralizadas de España se dan en las estribaciones de la Sierra de Guadarrama, en el Sistema Central (aguas de Bezoya y Fonsana) y las más mineralizadas, en torno al Sistema Ibérico (Peñalara, en La Rioja; Fuentecabras, en Zaragoza; Agua de Cardó, en Tarragona; y Font Lys y Aguas de Sierra Sanchís, en Valencia).

2. Las aguas más duras, por sus altos contenidos en sales cálcicas (asociados también a altos contenidos en sales magnésicas) son las de Insalus (Guipúzcoa), Peñaclara (La 
Tabla 1. Origen y composición de las aguas minerales naturales españolas envasadas

\begin{tabular}{|c|c|c|c|c|c|c|c|c|c|c|}
\hline Denominación & Lugar & $\mathrm{HCO}_{3}$ & $\mathrm{SO}_{4}{ }^{2-}$ & $\mathrm{Cl}^{-}$ & $\mathrm{NO}_{3}^{-}$ & $\mathrm{Na}^{+}$ & $K^{+}$ & $\mathrm{Mg}^{2+}$ & $\mathrm{Ca} a^{2+}$ & $\mathrm{SiO}_{2}$ \\
\hline Agua de Mondariz & $\begin{array}{l}\text { Balneario de Mondáriz, } \\
\text { Mondáriz }\end{array}$ & 164 & - & 17 & 一 & 49 & 3 & 6 & 10 & 13 \\
\hline Fuente del Val & Manantial de Mondáriz & 136 & 4 & 18 & - & 28 & 3 & 7 & 25 & - \\
\hline Fonteval & Pontevedra & & & & & & & & & \\
\hline \multirow[t]{3}{*}{ Cabreiroá } & Verín Orense & & & & & & & & & \\
\hline & 1988 & 408 & - & 9 & - & 144 & 12 & 3 & 8 & 37 \\
\hline & 1993 & 208 & - & 7 & - & 91 & 6 & 3 & 8 & 37 \\
\hline \multirow[t]{3}{*}{ Aguas de Sousas } & Verín Orense & & & & & & & & & \\
\hline & 1989 & 355 & - & 2 & 一 & 118 & - & - & - & 61 \\
\hline & 1994 & 236 & - & - & 一 & 82 & 5 & 2 & 5 & 53 \\
\hline Fontoira & $\begin{array}{l}\text { Manantial de Fontoira } \\
\text { Cospeiro, Lugo }\end{array}$ & 143 & 6 & 13 & - & 9 & - & 8 & 38 & 22 \\
\hline Fontecelta & $\begin{array}{l}\text { Manantial de Celtigos-Sarriá } \\
\text { Lugo }\end{array}$ & 240 & - & 58 & - & 125 & 4 & - & 11 & 49 \\
\hline Fuensanta & $\begin{array}{l}\text { Manantial de Fuensanta de } \\
\text { Buyeres, Srra. De Peña Mayor, } \\
\text { Nava Asturias }\end{array}$ & 262 & 91 & 12 & - & 24 & 7 & 14 & 84 & 67 \\
\hline Super-Quest & Manantial de Srra de Ques & 5 & 1 & 7 & - & 4 & - & 1 & 1 & - \\
\hline $\begin{array}{l}\text { Solfeguera } \\
\text { Agua de Cuevas }\end{array}$ & $\begin{array}{l}\text { Infiesto, Asturias } \\
\text { Srra. Fuentes de Invierno, }\end{array}$ & 239 & 13 & 3 & - & 1,5 & - & 25 & 47 & - \\
\hline Agua de Luevas & Puerto de San Isidro, Asturias & & & & & & & & & \\
\hline Corconte & $\begin{array}{l}\text { Balneario de Corconte, } \\
\text { Cantabria }\end{array}$ & 74 & 31 & 376 & - & 229 & 4 & 6 & 30 & 7 \\
\hline Solares & $\begin{array}{l}\text { Manantial de Fuencaliente, } \\
\text { Solares, Cantabria }\end{array}$ & 238 & 34 & 145 & 一 & 86 & 2 & 15 & 74 & 10 \\
\hline Insalus & $\begin{array}{l}\text { Manantial de Lizartza, } \\
\text { Guipuzcoa }\end{array}$ & 154 & 317 & 14 & - & 10 & - & 20 & 152 & 9 \\
\hline Betelú & Balneario de Betelú, Navarra & 221 & 79 & 242 & 3 & 151 & 2 & 17 & 84 & - \\
\hline Uralai & $\begin{array}{l}\text { Balneario de Belascoain, } \\
\text { Navarra }\end{array}$ & 132 & 17 & 351 & 一 & 157 & - & 15 & 56 & 3 \\
\hline Fontecabras & $\begin{array}{l}\text { Balneario de la Virgen, } \\
\text { Jaraba, Zaragoza }\end{array}$ & 296 & 130 & 61 & - & 一 & - & 40 & 95 & - \\
\hline El Cañar & $\begin{array}{l}\text { El Cañar 2, Puente de la Vega, } \\
\text { Jaraba, Zaragoza }\end{array}$ & 293 & 137 & 67 & - & - & - & 39 & 104 & 一 \\
\hline Veri & $\begin{array}{l}\text { Valle de Benasque, San Martín } \\
\text { De Veri-Bisaurri, Huesca }\end{array}$ & 196 & 18 & 6 & - & 13 & - & 6 & 58 & 2 \\
\hline $\begin{array}{l}\text { Ribagorza o } \\
\text { Aguas de Graus }\end{array}$ & $\begin{array}{l}\text { Manantial May } \\
\text { (Pirineo), Graus, Huesca }\end{array}$ & 331 & 18 & 31 & - & 24 & - & 27 & 67 & 12 \\
\hline Font del Pi & Guissona, Lleida & 317 & 207 & 17 & - & 27 & - & 66 & 67 & 42 \\
\hline Fonter & $\begin{array}{l}\text { Manantial Fonter, } \\
\text { Amer, Girona }\end{array}$ & 100 & 17 & 11 & - & 10 & 9 & 24 & - & - \\
\hline Font Picant & Amer, Girona & 67 & 5 & 1 & - & 6,5 & - & 11,5 & 5 & - \\
\hline Font del Regas & $\begin{array}{l}\text { Arbúcies (Montseny) } \\
\text { Girona }\end{array}$ & 112 & 8 & 6 & - & 12 & - & 3 & 29 & - \\
\hline Fontdor & $\begin{array}{l}\text { Sant Hilari, Sacalm, } \\
\text { Girona }\end{array}$ & 69 & 12 & 6 & - & 6 & - & 3 & 21 & 17 \\
\hline Font Vella & $\begin{array}{l}\text { Font Vella, Sant Hilari, } \\
\text { Sacalm, Girona }\end{array}$ & 153 & 14 & 11 & - & 13 & - & 8 & 41 & - \\
\hline Font Selva & $\begin{array}{l}\text { Manantial Font Selva, } \\
\text { Sant Hilari, Sacalm, Girona }\end{array}$ & 229 & 10 & 7 & - & 16 & 一 & 9 & 51 & 22 \\
\hline
\end{tabular}




\begin{tabular}{|c|c|c|c|c|c|c|c|c|c|c|}
\hline Denominación & Lugar & $\mathrm{HCO}_{3}$ & $\mathrm{SO}_{4}^{3-}$ & $\mathrm{Cl}$ & $\mathrm{NO}_{3}$ & $\mathrm{Na}^{+}$ & $K^{+}$ & $\mathrm{Mg}^{2+}$ & $\mathrm{Ca} a^{2+}$ & $\mathrm{SiO}_{2}$ \\
\hline Vichy Catalán & $\begin{array}{l}\text { M. Vichy, Caldes de } \\
\text { Melavella, Girona }\end{array}$ & 213 & 5 & 60 & - & 111 & - & 5 & - & 8 \\
\hline \multirow[t]{4}{*}{$\begin{array}{l}\text { Agua de Viladrau } \\
\text { o Fontalegre }\end{array}$} & $\begin{array}{l}\text { Manantial Fontalegre, } \\
\text { La Cuadanya, Viladray, Girona }\end{array}$ & & & & & & & & & \\
\hline & 1988 & 42 & 8 & 5 & - & 8 & - & 2 & 13 & 24 \\
\hline & 1991 & 68 & 8 & 8 & - & 8 & - & 3 & 20 & 21 \\
\hline & 1994 & 91 & 7 & 8 & - & 9 & - & 3 & 26 & 22 \\
\hline $\begin{array}{l}\text { Agua del Valle } \\
\text { De Cardó }\end{array}$ & $\begin{array}{l}\text { Balneario de Cardó, } \\
\text { Benifallet, Tarragona }\end{array}$ & 387 & 19 & 18 & - & 7 & - & 35 & 81 & 4 \\
\hline Font Lys & $\begin{array}{l}\text { Manantial Font-Lys, } \\
\text { Manuel, Valencia }\end{array}$ & 329 & 142 & 145 & - & 94 & - & 36 & 112 & - \\
\hline \multirow[t]{2}{*}{ Font Sol } & El Moreral, Aguas de & - & 248 & - & - & - & 3 & 48 & 81 & 5 \\
\hline & $\begin{array}{l}\text { Srra. Sanchis } \\
\text { Fuente la Higuera, Valencia }\end{array}$ & 239 & 239 & - & - & - & 3 & 51 & 118 & - \\
\hline Sierra Sanchís & Fuente la Higuera, Valencia & 268 & 259 & 129 & - & 78 & - & 50 & 122 & 13 \\
\hline Fuente Primavera & Requena, Valencia & 295 & 295 & 43 & - & 21 & - & 23 & 87 & 7 \\
\hline \multirow[t]{4}{*}{ Peñaclara } & $\begin{array}{l}\text { Torrecilla en Cameros, } \\
\text { La Rioja }\end{array}$ & & & & & & & & & \\
\hline & 1987 & 245 & 300 & 20 & - & 16 & 2 & 38 & 155 & - \\
\hline & 1991 & 199 & 335 & 14 & - & 13 & 1 & 34 & 134 & - \\
\hline & 1996 & 227 & 273 & 15 & - & 14 & 1 & 28 & 141 & - \\
\hline Monte Pinos & Almazán. Soria & 287 & 1,5 & 5 & - & 2 & - & 3 & 92 & - \\
\hline Carrizal & $\begin{array}{l}\text { S. Andrés de Rabanedo } \\
\text { León }\end{array}$ & 104 & 2 & 3 & - & 1 & - & 6 & 27 & 9 \\
\hline San Andrés & $\begin{array}{l}\text { S. Andrés de Rabanedo } \\
\text { León }\end{array}$ & 74 & 2 & 3 & - & 1 & - & 7 & 17 & 6 \\
\hline Santolín & Quintana-Urria, Burgos & 270 & 6 & 5 & - & 2 & - & 2 & 89 & - \\
\hline Castrovita & Castromonte, Valladolid & 249 & 7 & 13 & - & 7 & - & 13 & 72 & 20 \\
\hline Bezoya & $\begin{array}{l}\text { Ortigosa del Monte, } \\
\text { Segovia }\end{array}$ & 11 & - & 1 & 2 & 2 & - & - & 2 & 7 \\
\hline $\begin{array}{l}\text { De la Platina } \\
(\text { («rechs») }\end{array}$ & Salamanca & 79 & 18 & 16 & - & 9 & - & 16 & 16 & 10 \\
\hline Babilafuente & $\begin{array}{l}\text { Fte. Del Caño Babilafuente } \\
\text { Babilafuente, Salamanca }\end{array}$ & 167 & 6 & 6 & - & 14 & - & 5 & 42 & 64 \\
\hline Fonsana & $\begin{array}{l}\text { La Cabrera (Somosierra) } \\
\text { Madrid }\end{array}$ & 38 & 3 & 5 & - & 7 & - & - & 7 & 34 \\
\hline Agua del Rosal & Calera y Chozas, Toledo & 289 & 7 & 41 & - & 45 & - & 14 & 64 & 39 \\
\hline Valtorre & $\begin{array}{l}\text { Manantial Valtorre, Belvis } \\
\text { De la Jara, Toledo }\end{array}$ & 191 & - & 40 & 4 & 31 & - & 24 & 26 & - \\
\hline Fontemilla & $\begin{array}{l}\text { Reserva de Cutamilla, } \\
\text { Sigüenza, Guadalajara }\end{array}$ & 300 & - & - & - & 4 & - & 24 & 78 & - \\
\hline Fuente Liviana & $\begin{array}{l}\text { Huerta del Marquesado } \\
\text { Cuenca }\end{array}$ & 254 & 16 & 2 & 1 & - & 0,5 & 19 & 68 & - \\
\hline Solan-de Cabras & $\begin{array}{l}\text { Balneario Solan-de Cabras, } \\
\text { Beteta, Cuenca }\end{array}$ & 280 & 17 & 9 & - & 5 & 1 & 25 & 60 & 8 \\
\hline Los Riscos & $\begin{array}{l}\text { Los Riscos de la Higuera } \\
\text { Alburquerque, Badajoz }\end{array}$ & 11 & - & 23 & - & 16 & 2 & 2 & 4 & - \\
\hline Neval & Moratalla, Murcia & 243 & 120 & - & - & - & - & 45 & 58 & - \\
\hline Lanjarón & $\begin{array}{l}\text { Lanjarón, Sierra Nevada, } \\
\text { Granada }\end{array}$ & 123 & 24 & - & - & - & 1 & 11 & 33 & - \\
\hline San Vicente & $\begin{array}{l}\text { Balneario de Lanjarón, } \\
\text { Lanjarón, Granada }\end{array}$ & 81 & 19 & 3 & - & 6 & - & 7 & 22 & - \\
\hline Zambra & $\begin{array}{l}\text { Nacimiento del Zanbra } \\
\text { Rute, Córdoba }\end{array}$ & 140 & - & - & - & 22 & 1 & 16 & 65 & - \\
\hline
\end{tabular}


Rioja), Font Sol y Sierra Sanchís (Valencia). El contenido en sulfatos es especialmente alto en estas aguas y también en las de Lleida, Zaragoza y Murcia.

3. Las aguas minerales con mayor contenido en sílice son las de Lugo y Orense, en Galicia; Nava, en Asturias; y algunas de Girona, en Cataluña. También la de La Cabrera, en Madrid, es alta en $\mathrm{SiO}_{2}$.

4. Las aguas con mayor contenido en potasio son también las de Lugo, Orense, Nava y algunas de Girona. Asimismo, Corconte exhibe contenidos moderados de este ion.

5. Las aguas que experimentan mayor variación en su composición son las de Cabreiró, Sousas y Viladrau: los contenidos en bicarbonato sódico, para las dos primeras, y de bicarbonato cálcico, para la segunda, varían de unos años a otros hasta en un $40 \%$ respecto al valor medio. De 1988 a 1993 el contenido en bicarbonato sódico del agua Cabreiroá descendió en un 46\%. En 1994, el agua de Sousas alcanzó su contenido mínimo en bicarbonato sódico (un 20\% menos de la media) mientras el agua de Viladrau alcanzaba su contenido máximo en bicarbonato cálcico de la última década (un 40\% por encima de la media). Entre 1987 y 1991 también se produjo un descenso significativo de la salinidad del agua Peñaclara de Torrecilla en Cameros (La Rioja).

6. Si comparamos la composición de dos aguas con localizaciones próximas (s.c., presumiblemente filtradas a través de formaciones minerales del subsuelo muy similares), la situada más al sur resulta más salina en $\mathrm{NaCl}$ que la ubicada más al norte. Por ejemplo, en Lugo, Fontecelta (Céltigos) es más salina que Fontoira (Cospeito); en Huesca, Graus más que Veri-Bisaurri; y en Girona, Font del Regas que Viladrau. La constatación de esta observación se produce hasta en el caso de localizaciones como Corconte y Solares, cuando por la ubicación de la primera, en el interior y próxima a un embalse, cabría esperar menor salinidad que el agua de una población como Solares, próxima a la costa.

7. Si la comparación de la composición de aguas con localizaciones próximas se realiza en la dirección izquierda-derecha y entre ambas no se interpone algún accidente geográfico importante, resulta que el agua de la localización a la izquierda está más mineralizada que la de la derecha. Esta observación puede ser ejemplificada con las aguas de Amer, Girona y Asturias. La situación es drástica para el caso de las aguas de Nava (Fuensanta) e Infiesto (Quess): la de Nava es una de las más mineralizadas de España, mientras la de Infiesto es una de las menos mineralizadas del territorio peninsular.

8. Además de las coordenadas de longitud y latitud, la altitud/profundidad del afloramiento/acuífero también parece jugar un papel importante en cuanto a la composición del agua. En el caso de los manantiales de Fontdor y Font Selva, ambos en el mismo término municipal (Sant Hilari Sacalm) pero con diferentes 
niveles de nacimiento y/o captación (a $1000 \mathrm{~m}$ de altura el primero y a $200 \mathrm{~m}$ de profundidad el segundo), el contenido en bicarbonatos es muy diferente y mayor para captaciones profundas (caso de Font Selva). Si la comparación se realiza entre las aguas Veri y Ribagorza, que brotan también a diferentes alturas (a 1235 $m$ en el caso de Veri y a una altura inferior en el caso de Ribagorza) y desde diferentes profundidades (en el de Ribagorza, desde $1800 \mathrm{~m}$ ), la mayor concentración en bicarbonatos sigue correspondiendo al agua situada a más bajos niveles, o sea, al agua de Ribagorza o de Graus. En el caso del agua de San Andrés de Rabanedo, en León, las captaciones a similar profundidad (250` $50 \mathrm{~m}$ ) para Carrizal y San Andrés, proporcionan contenidos en bicarbonatos similares (90`15 $\mathrm{mg} / \mathrm{L})$.

\section{DISCUSIÓN}

Aunque cada una de las asociaciones referidas en el trabajo ha sido justificada, la casuística aportada es insuficiente para conceder a tales asociaciones un carácter general. En todos los casos se precisa comprobación de los resultados con los que aporten estudios paralelos sobre lugares más o menos alejados de la Península Ibérica. Este requerimiento es especialmente importante para el caso de la asociación salinidad-longitud geográfica, pues su generalización implicaría responsabilizar al sentido de rotación de la Tierra (oeste a este) en la creación de gradientes de concentración salina decreciente entre acuíferos vecinos, con una acumulación salina en los más occidentales. Adicionalmente, la constatación general de las asociaciones salinidad-longitud y mineralización-latitud sería acorde con la existencia de una deriva salina hacia el S-O, responsable, entre otros factores, del carácter hipertónico de las aguas minerales (solo útiles en balneación) de Huelva y Cádiz (por ejemplo, Fuente Amarga, en Chiclana) y, a escala mundial, del carácter salino de los desiertos de Sonora, Patagonia, Namibia y Gran Desierto Victoria.

\section{BIBLIOGRAFÍA}

IÑIGO, J.M. y ARADILLAS, A. (1996): Balnearios de España, Ed. Everest, S.A., León. SÁNCHEZ DE LA MUELA y GONZÁLEZ PARRA, citados en: http://www.citelan.es/solan/esp/estudio.html 


\section{APÉNDICE: SOBRE LAS AGUAS MINERALES NATURALES DE PORTUGAL}

La prosecución del estudio sobre las aguas minerales naturales de España con el recientemente realizado sobre las ngias de nascente de Portugal, nos ha llevado a resultados que confirman las conclusiones previamente alcanzadas. Así, la observación de que las aguas más meridionales de España son más salinas en $\mathrm{NaCl}$ que las septentrionales resulta también válida para las aguas portuguesas: los contenidos en iones cloruro y sodio de las aguas Cruzeiro y Sno Silvestre aparecen más altos que los de las aguas que emergen de las sierras de Caramulo y da Estrela, y estos, aún más que los de las aguas norteñas de la Serra do Gerês (ngua do Fastio) y Tras-os-Montes (Caraalhelhos). Por otra parte, el hallazgo de composiciones más salinas para las aguas españolas occidentales respecto a las orientales adquiere generalización a nivel peninsular con la evidencia de que las aguas portuguesas de Caramulo, Serrana y Luso, que nacen más al oeste que las de la Serra da Estrela, se presentan más concentradas en sales que las de esta localización; o con las aguas de Sao Silvestre y Vitalis, ésta en la sierra de S. Mamede (Vitalis) y aquélla, más al oeste.

En el capítulo de discusión, la aparente contradicción que parece desprenderse de la comparación de las concentraciones de las aguas Do Fastio y Carvalhelhos y la ubicación relativa de sus manantiales, resulta modulada por la constatación, ya hecha observar para las aguas españolas, que la interposición de un accidente geográfico notable entre ellos (en este caso el río Zézere) invalida cualquier comparación que pudiera resultar. La alta concentración en $\mathrm{HCO}_{3}{ }^{-}$del agua de Boticas (Corvalhelhos) resulta, por otra parte similar al de localizaciones españolas de la misma latitud: de hecho, es intermedia entre la de Castrovita, de Valladolid, y La Platina, de Salamanca.

Tabla 2. Origen y composición de aguas minerales naturales portuguesas envasadas

\begin{tabular}{|c|c|c|c|c|c|c|c|c|c|c|c|c|}
\hline Denominación & Lugar & $\mathrm{HCO}_{3-}$ & $\mathrm{SO}_{4}^{2-}$ & $\mathrm{Cl}^{-}$ & $\mathrm{NO}_{3}^{-}$ & $N n^{+}$ & $K^{+}$ & $\mathrm{Mg}^{2+}$ & $\mathrm{Cn}^{2+}$ & $\mathrm{SiO}_{2}$ & RS/MT & $p H$ \\
\hline Do Fastio & $\begin{array}{l}\text { Serra do Gerês, } \\
\text { Terras de Bouro }\end{array}$ & 9 & - & 5 & - & 5 & 0,6 & - & 2 & 13 & 34 & 5,9 \\
\hline Carvalhelhos & $\begin{array}{l}\text { Fntes. Lucy y Stella } \\
\text { Carvalhelhos Boticas } \\
\text { Trasos-Montesa }\end{array}$ & 134 & - & 3 & - & 50 & - & - & - & 39 & 246 & 7,9 \\
\hline Ladeira & Ladeira de Envendos & 2 & - & 7 & - & 4 & - & - & 1 & 11 & 28 & 5,0 \\
\hline Serrana & $\begin{array}{l}\text { Fuente do Cabril } \\
\text { Serra do Caramulo, } \\
\text { Agueda }\end{array}$ & 13 & - & $<5$ & - & 7 & - & - & - & 14 & 44 & 5,6 \\
\hline Caramulo & $\begin{array}{l}\text { Varzielas, Olivera } \\
\text { de Frades }\end{array}$ & 31 & - & 8 & 1,4 & 13 & - & - & 4 & 26 & 94 & 6,3 \\
\hline Serra da Estrela & $\begin{array}{l}\text { Fuente Vidoeira } \\
\text { Cabeça do Velho } \\
\text { Gouveia }\end{array}$ & 9 & - & 2,5 & - & 3 & - & - & 1 & - & 24 & 6,0 \\
\hline \multirow[t]{3}{*}{ Vitalis } & $\begin{array}{l}\text { Luso } \\
\text { Serra de Sao Mamede } \\
\text { Castelo de Vide }\end{array}$ & 9 & 1,6 & 9 & 1,9 & 6,3 & - & 1,3 & 1 & 13 & 44 & 5,9 \\
\hline & 1992 & 8,5 & 1,5 & 11 & 0,9 & 6 & 2 & 0,4 & 1 & $>25$ & 48 & 5,6 \\
\hline & 1998 & 5 & 2,4 & 7 & 2,5 & 5,5 & 2 & 0,5 & 1 & 18 & 43 & 5,6 \\
\hline Sao Silvestre & Pernes Santarém & 145 & - & 34 & - & 30 & - & - & 31 & - & 200 & 7,3 \\
\hline Cruzeiro & Cruzeiro & 122 & 1,7 & 17 & 1 & 10 & 6 & 12 & 18 & 15 & 141 & 6,9 \\
\hline
\end{tabular}

